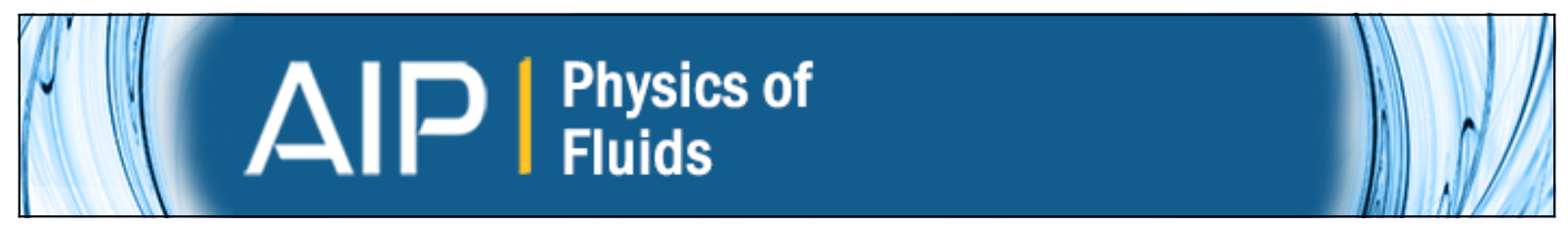

\title{
On the atmosphere of a moving body
}

Johan Roenby and Hassan Aref

Citation: Physics of Fluids (1994-present) 22, 057103 (2010); doi: 10.1063/1.3406960

View online: http://dx.doi.org/10.1063/1.3406960

View Table of Contents: http://scitation.aip.org/content/aip/journal/pof2/22/5?ver=pdfcov

Published by the AIP Publishing

Copyright by the American Institute of Physics. On the atmosphere of a moving body

Roenby, Johan and Aref, Hassan, Physics

of Fluids (1994-present), 22, 057103

(2010), DOI:http://

dx.doi.org/10.1063/1.3406960

\section{AlP Re-register for Table of Content Alerts}




\title{
On the atmosphere of a moving body
}

\author{
Johan Roenby ${ }^{\mathrm{a})}$ and Hassan Aref ${ }^{\mathrm{b})}$ \\ Center for Fluid Dynamics, Technical University of Denmark, Kgs. Lyngby DK-2800, Denmark
}

(Received 21 December 2009; accepted 29 March 2010; published online 11 May 2010)

\begin{abstract}
We explore whether a rigid body moving freely with no circulation around it in a two-dimensional ideal fluid can carry a fluid "atmosphere" with it in its motion. Somewhat surprisingly, the answer appears to be "yes." When the body is elongated and the motion is dominated by rotation, we demonstrate numerically that, indeed, regions of fluid follow the body in its motion. We see this as an example of the stability of Kolmogorov-Arnold-Moser tori. These observations of an atmosphere around a moving body with no circulation around it appear to be new. () 2010 American Institute of Physics. [doi:10.1063/1.3406960]
\end{abstract}

\section{INTRODUCTION}

By the atmosphere of a moving body we mean a region of fluid (area or volume) that moves alongside the body, as if bound to it, in its motion through the fluid. In this sense the notion of an atmosphere was probably first used by Kelvin ${ }^{1}$ in connection with the fluid volume carried forward with a vortex pair or vortex ring. Translating vortex patterns generally carry fluid with them. Here we are interested in a related, even more immediate problem: if we launch a body on a trajectory through fluid otherwise at rest, will it carry a region of fluid with it?

In a viscous fluid the answer would have to be "yes." For example, consider a circular cylinder translating uniformly. For a range of moderate Reynolds numbers the flow is stationary when viewed from the reference frame moving with the cylinder and a vortex pair exists behind the cylinder. The fluid inside the separatrix enclosing this vortex pair may be regarded as an atmosphere since it follows the body in its motion. If the fluid is inviscid and we allow a finite circulation around a uniformly translating cylinder, we may also have a constant separatrix around the cylinder when viewed in a comoving frame. Again we may regard the fluid inside this separatrix as an atmosphere of the moving body since its constituent fluid particles stay with the body forever. But what if the fluid is inviscid and there is no vorticity and no circulation around the body? Can fluid particles then be carried along with the body in its motion or are they, so to speak, washed away as the body proceeds? Certainly for simple translational motion of the body, as analyzed by Maxwell ${ }^{2}$ for a translating circular cylinder with no circulation around it, only the two particles sitting at the forward and rearward stagnation points can be said to be carried along forever. We would hardly consider these particles, even if augmented by the incoming forward stagnation streamline, to be an atmosphere. For pure translation of a noncircular body the flow is simply obtained from the

\footnotetext{
a) Also at Department of Mathematics, Technical University of Denmark, Kgs. Lyngby DK-2800, Denmark. Electronic mail: johan.roenby@gmail.com.

b) Also at Department of Engineering Science and Mechanics, Virginia Tech, Blacksburg, VA 24061, USA.
}

circular case by a conformal mapping. Thus, for simple translational motion of a body in an inviscid fluid the answer to our question would be "no." Our notion of an atmosphere implies areas or regions of fluid of finite area. Points, curves, and other "sets of measure zero" do not constitute an atmosphere.

In general, however, a noncircular body moving freely in an inviscid fluid in response to the pressure force on its boundary both translates and rotates. In the two-dimensional (2D) case, to which we confine attention, the motion of the body is integrable. For a symmetric body shape, such as an ellipse, the motion may be obtained in terms of elliptic integrals as first shown by Kirchhoff. ${ }^{3}$ For such a body there are three "eigenmotions," namely, uniform translation along each of the axes of symmetry and uniform rotation around the center of symmetry. In the case of pure rotation, the stream function in the body-fixed, corotating frame of reference may be found. For sufficiently elongated body shapes it clearly shows islands of fluid corotating with the body, each island surrounding an elliptical fixed point. This is discussed in Ref. 3, Sec. 72, where the important 1913 paper by Morton ${ }^{4}$ is mentioned. Forty years later, the phenomenon was rediscovered by Darwin ${ }^{5}$ in connection with his analysis of what is now called drift. Dynamical systems theory, in particular the Kolmogorov-Arnold-Moser (KAM) theorem ${ }^{6}$ then shows that such islands are stable to small perturbations. Hence, we expect that for small translational velocity (relative to the angular velocity times a typical dimension of the body), the islands found for the case of pure rotation will be partially stable, in the sense that there will still be an area of fluid that follows the body in its motion. This area constitutes the atmosphere.

In the remainder of the paper we document the statements made here. In Sec. II we provide some details for the analytically accessible case of pure rotation. In Sec. III we then explore by numerical experiments how the stability and eventual breakdown of the atmosphere depends on initial conditions. Finally, Sec. IV contains our conclusions. 


\section{EQUATIONS OF MOTION AND ANALYTICAL SOLUTIONS}

We think of the plane of motion as the complex plane and denote the instantaneous position of the geometrical center of the body by $z_{0}(t)$ and its instantaneous orientation by $\theta(t)$. Calling a general point in the plane in laboratory coordinates $z$, we may define body-fixed coordinates by $\tilde{z}=\left(z-z_{0}\right) e^{-i \theta}$. By the Riemann mapping theorem any body shape in the $\tilde{z}$-coordinates may be obtained by a conformal mapping $f(\zeta)$ of the unit circle in the complex $\zeta$-plane. Here we consider body shapes obtainable from the Joukowski mapping,

$$
\tilde{z}=f(\zeta)=\zeta+\frac{a^{2}}{\zeta-\zeta_{0}},
$$

where the parameter $0 \leq a \leq 1$, and $\zeta_{0}$ is complex. To avoid self-crossing of the body contour, $f(|\zeta|=1)$, we must have $\left|\zeta_{0} \pm a\right| \leq 1$. When $\zeta_{0}=0$ the body contour is an ellipse, the special cases $a=0$ and $a=1$ corresponding, respectively, to a circle and a flat plate connecting $\tilde{z}= \pm 2$. Setting $\zeta_{0} \neq 0$ introduces an asymmetry of the body. The effect of this asymmetry on the atmosphere is also of interest.

The linear and angular velocity of the body will be called $v_{0}(t)=\dot{z}_{0}(t)$ and $\omega(t)=\dot{\theta}(t)$, respectively. The complex conjugate of the fluid velocity is given by

$$
\overline{u(z, t)}=e^{-i \theta(t)} w^{\prime}(\zeta, t) / f^{\prime}(\zeta),
$$

where the bar denotes complex conjugation and the prime denotes differentiation with respect to $\zeta$. The $\zeta$-coordinate complex potential $w(\zeta, t)$ associated with the body motion may be obtained by a method similar to that described by Milne-Thomson, ${ }^{7}$ Sec. 9.63. For body shapes obtained from Eq. (1) the result is

$$
\begin{aligned}
w(\zeta, t)= & \left(-\frac{1}{\zeta}+\frac{a^{2}}{\zeta-\zeta_{0}}\right) U(t)-i\left(\frac{1}{\zeta}+\frac{a^{2}}{\zeta-\zeta_{0}}\right) V(t) \\
& -\frac{i a^{2}}{\zeta-\zeta_{0}}\left(\frac{1}{\zeta}+\frac{\zeta_{0} a^{2}}{1-\left|\zeta_{0}\right|^{2}}\right) \omega(t) .
\end{aligned}
$$

Here we have defined the components of the tilted velocity, $U(t)+i V(t)=v_{0}(t) e^{-i \theta(t)}$. We are particularly interested in the fluid motion relative to the body. This is obtained by subtracting $v_{0}(t)+i \omega(t)\left[z-z_{0}(t)\right]$ from $u(z, t)$ in Eq. (2) and then multiplying the result by $e^{-i \theta(t)}$.

The equations of motion for the body may be obtained by using conservation of the linear impulse $P$ and angular impulse $L$ of the system. This gives the body equations of motion,

$$
\dot{z}_{0}(t)=[U(t)+i V(t)] e^{i \theta(t)}, \quad \dot{\theta}(t)=\omega(t),
$$

with $U, V$, and $\omega$ given by

$$
\left(\begin{array}{c}
U \\
V \\
\omega
\end{array}\right)=M^{-1}\left(\begin{array}{c}
\operatorname{Re}\left(P e^{-i \theta(t)}\right) \\
\operatorname{Im}\left(P e^{-i \theta(t)}\right) \\
L-\operatorname{Im}\left[\overline{z_{0}(t)} P\right]
\end{array}\right) .
$$

Here $M$ is the effective mass tensor,

$$
M=\left(\begin{array}{ccc}
m+A_{11} & 0 & A_{13} \\
0 & m+A_{22} & A_{23} \\
A_{13} & A_{23} & I_{0}+A_{33}
\end{array}\right),
$$

which is symmetric and constant in time. The matrix $M$ depends on the body mass $m$ and moment of inertia $I_{0}$ (with respect to $z_{0}$ ). For body shapes obtained from Eq. (1) these are, respectively,

$$
\begin{aligned}
& m=\rho_{b} \pi\left(1-\epsilon^{2}\right), \\
& I_{0}=\frac{\rho_{b} \pi}{2}\left[1-\epsilon^{4}-2\left(1+\epsilon^{2}\right)\left|\zeta_{0}\right|^{2} \epsilon^{2}-4 \operatorname{Re}\left(\zeta_{0}^{2}\right) \epsilon^{3}\right],
\end{aligned}
$$

where $\rho_{b}$ is the density of the homogeneous body. (We have chosen our unit of mass such that the fluid density is 1.) The parameter $\epsilon=a^{2} /\left(1-\left|\zeta_{0}\right|^{2}\right)$. Further, $M$ depends on the elements of the added mass tensor defined by

$$
A_{j k}=-\frac{1}{2 i} \oint_{|\zeta|=1} \overline{w_{j}(\zeta)} \frac{\mathrm{d} w_{k}(\zeta)}{\mathrm{d} \zeta} \mathrm{d} \zeta, \quad j, k=1,2,3 .
$$

In this formula $w_{1}, w_{2}$, and $w_{3}$ are the complex unit potentials associated with the body motion. They are, respectively, the prefactors of $U, V$, and $\omega$ in Eq. (3). Due to the symmetry one may always choose body-fixed coordinates such that $A_{12}=0$. For the Joukowski body shapes described by Eq. (1) one obtains (e.g., by application of the area theorem of Milne-Thomson, ${ }^{7}$ Sec. 5.43) the remaining added mass tensor elements

$$
\begin{aligned}
& A_{11}=(1-\epsilon)^{2}-2\left|\zeta_{0}\right|^{2}, \\
& A_{13}=-\operatorname{Im}\left(\zeta_{0}\right) \epsilon^{2}\left(3-2\left|\zeta_{0}\right|^{2}-\epsilon\right), \\
& A_{22}=(1+\epsilon)^{2}-2\left|\zeta_{0}\right|^{2}, \\
& A_{23}=\operatorname{Re}\left(\zeta_{0}\right) \epsilon^{2}\left(3-2\left|\zeta_{0}\right|^{2}+\epsilon\right), \\
& A_{33}=\epsilon^{2}\left\{\left(2-\left|\zeta_{0}\right|^{2}\right)\left[1+2 \operatorname{Re}\left(\zeta_{0}^{2}\right) \epsilon\right]+\left|\zeta_{0}\right|^{2} \epsilon^{2}\right\} .
\end{aligned}
$$

Noting that the values of the constants $P$ and $L$ for given initial body position, orientation, and linear and angular velocity may be obtained from Eq. (4b), we now have all we need to solve the problem numerically.

To test a numerical integration one may monitor the total energy of the system,

$$
E=\sum_{i, j} \frac{1}{2}(U, V, \omega)_{i} M_{i j}(U, V, \omega)_{j},
$$

which should be a conserved quantity. The existence of the four constants of the motion $P, L, E$ guarantees integrability of the body motion since the phase space is spanned by the six variables $\left(z_{0}, \theta, v_{0}, \omega\right)$. For a generalization of the equation of motions to the situation where point vortices are present in the fluid and there is a circulation around the body, see Ref. 8.

A homogeneous body moving in 2D ideal fluid has two axes of permanent translation, and it may also rotate without translation. For a homogeneous ellipse the axes of permanent translation are the geometrical major and minor axes. The pure rotation takes place around the center of the ellipse. For a body of general shape, including Joukowski airfoils, one 


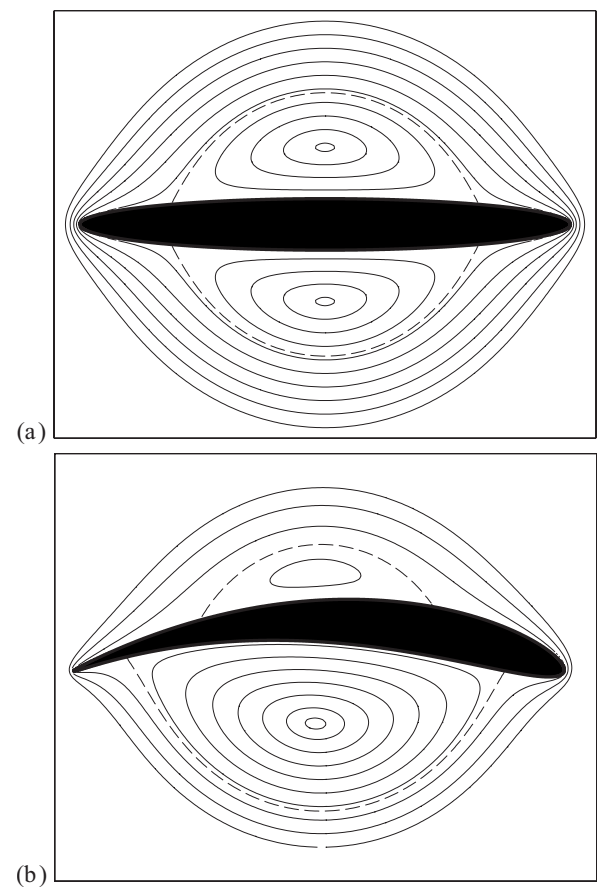

FIG. 1. Streamlines in the body-fixed frame for uniform rotation of (a) an elliptical cylinder with $a=0.9$ and (b) a Joukowski airfoil with $a=0.9$, $\zeta_{0}=-0.06-0.2 i$, cf. Eq. (1). Dashed lines are stagnation streamlines. The geometry of the streamline pattern depends on the mass of the airfoil but is invariant to mass variations for the symmetrical ellipse. Here the mass of the airfoil is ten times the mass of the fluid it displaces.

has to seek out the solution for which the linear impulse $P$ vanishes. The angular velocity of the body is then constant as is the translational velocity of the body in the body-fixed reference frame. Inserting $P=0$ in Eq. (4b), one easily finds the conditions for pure rotation, namely, $U=-\omega A_{13} / M_{11}$ and $V=-\omega A_{23} / M_{22}$. Since the motion $U+i V+i \omega \widetilde{z}$ may also be written $i \omega[\widetilde{z}-i(U+i V) / \omega]$, we have uniform rotation with constant angular velocity $\omega$ around the point which in bodyfixed coordinates is $A_{23} / M_{22}-i A_{13} / M_{11}$.

A contour plot of the stream function corresponding to the pure rotation solution for $a=0.9$ and $\zeta_{0}=0$ is shown in Fig. 1(a). If we consider the flow from the corotating frame of reference, it is steady and particle paths follow streamlines. The two regions surrounding the elliptic fixed points on either side of the body will follow it round as it rotates and, thus, constitute an atmosphere, albeit of a body rotating in place.

In Fig. 1(b) we show a streamline pattern for a uniformly rotating Joukowski airfoil (or, more precisely, a "near airfoil" shape). The profile is obtained by applying the conformal mapping of Eq. (1) to the unit circle again with $a=0.9$ but now with $\zeta_{0}=-0.06-0.2 i$. The steadily rotating case of the Joukowski airfoil is the solution of the equations of motion with vanishing linear impulse $P$. For a body that is very heavy compared with the fluid it displaces, the center of rotation in body-fixed coordinates, $A_{23} / M_{22}-i A_{13} / M_{11}$, will be close to 0 since the $M_{i j}$ 's (in contrast to the $A_{i j}$ 's) increase with the body density $\rho_{b}$. Thus, in order for the rotation to take place on a relatively small circle, we must use a body mass that is large compared with the mass of the fluid it displaces. In Fig. 1(b) this ratio has been set to 10. (For a symmetric shape, such as an ellipse, the center of rotation is the symmetry center and the steady streamline pattern in the corotating frame is independent of body mass.) For the parameters chosen the streamline pattern in Fig. 1(b) has the dual elliptic points with their surrounding regions that we then wish to perturb by adding a translation.

In these simple cases of pure rotation we have the luxury of being able to write formulas for the shape of the atmosphere, as did Kelvin ${ }^{1}$ for the translating vortex pair. Darwin ${ }^{5}$ described the motion in the following way: "When the trajectories are considered of the fluid surrounding a rotating body, it is shown that the fluid particles slowly drift round the body, even though the motion is irrotational and without circulation. There seems to be in some respects a closer resemblance between the behavior of the idealized hydrodynamic fluid and a real fluid than might be expected from the well-known discrepancies between them."

\section{NUMERICAL EXPLORATIONS}

As outlined in Sec. I we now wish to perturb the state of steady rotation by giving the cylinder a nonzero translation at the initial instant. The solutions for the body motion then take one of two forms, with a crossover "separatrix" motion dividing them. ${ }^{3}$ For small initial velocity the body continues to rotate end-over-end as it translates. For falling bodies this kind of motion has been called tumbling, and we will use this term here as well. As the initial velocity is increased, a point is reached where the forward motion is large enough for the body never to complete a full turn but for its orientation to "rock" back and forth. This kind of motion has been called fluttering (again for falling bodies). For falling bodies the sensitivity in switching between tumbling and fluttering is a key ingredient in producing chaotic motion. ${ }^{9}$ We have shown that a similar mechanism occurs in body-vortex interactions. ${ }^{8}$ In the present problem of the trapping of advected particles in an atmosphere, the tumbling motions turn out to be the most effective. This may be understood by appeal to the general ideas in the KAM theorem of dynamical systems theory. The elliptical islands seen in the unperturbed, steadyrotation problem in Fig. 1 have an intrinsic stability to perturbation. Whereas some of the orbits in the complete island structure will break down to chaos, the main structure will remain. It is this stable remnant that continues to constitute the atmosphere of the tumbling body.

The numerical experiments are quite simple: a number of particles are started in an array that sweeps through the initial positions of interest. Here we have chosen around 100 initial positions between $\pm 3 i$ on the imaginary axis of the $\zeta$-coordinates. For each return of the body to its initial orientation, $\theta=0$, the particle positions are plotted, as if illuminated by a stroboscope set to the period of rotation of the body. Runs are for a set time interval that translates into about 1000 revolutions of the body. The linear and angular impulses of the system are strictly conserved in our numerics, since these conservation laws are used as three of the equations of motion for the body. The numerical integration was performed with the MATLAB ${ }^{\circledR}$ ode45 solver with the rela- 
(a)

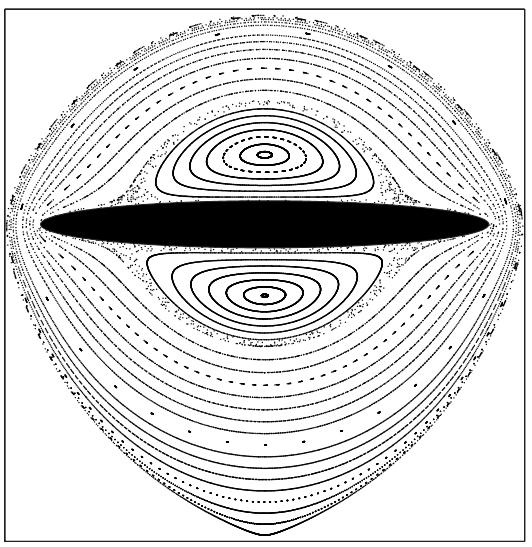

(d)
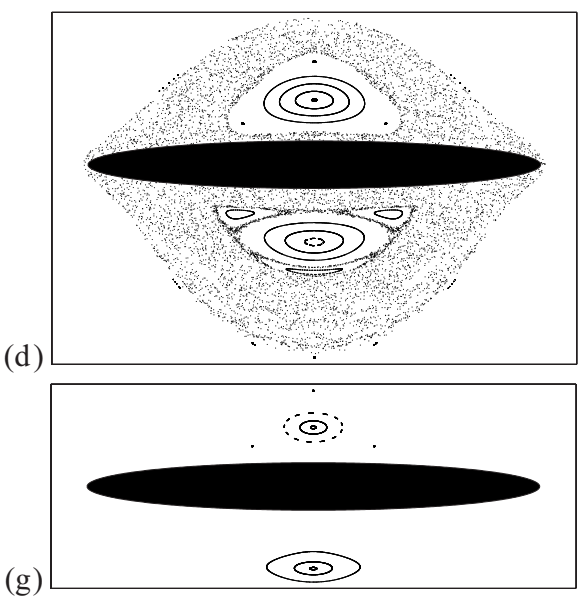

(b)

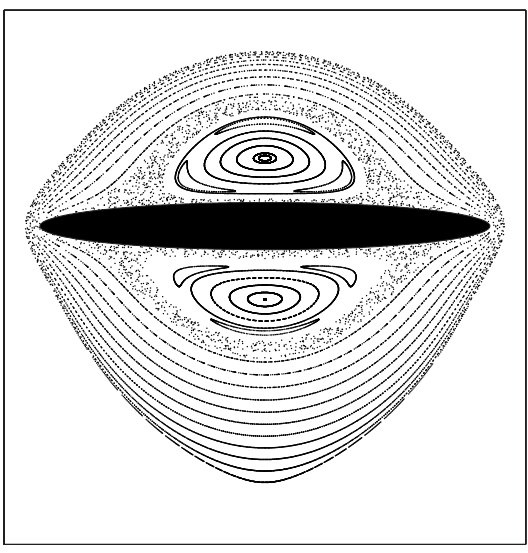

(e)

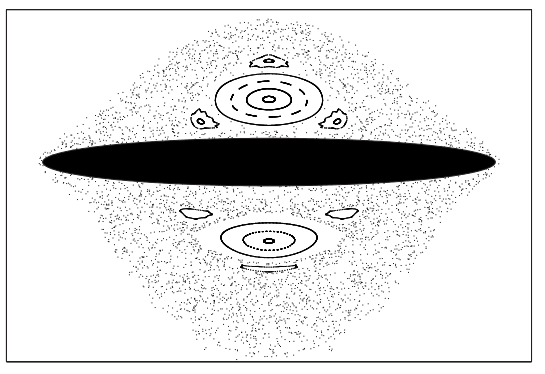

(c)
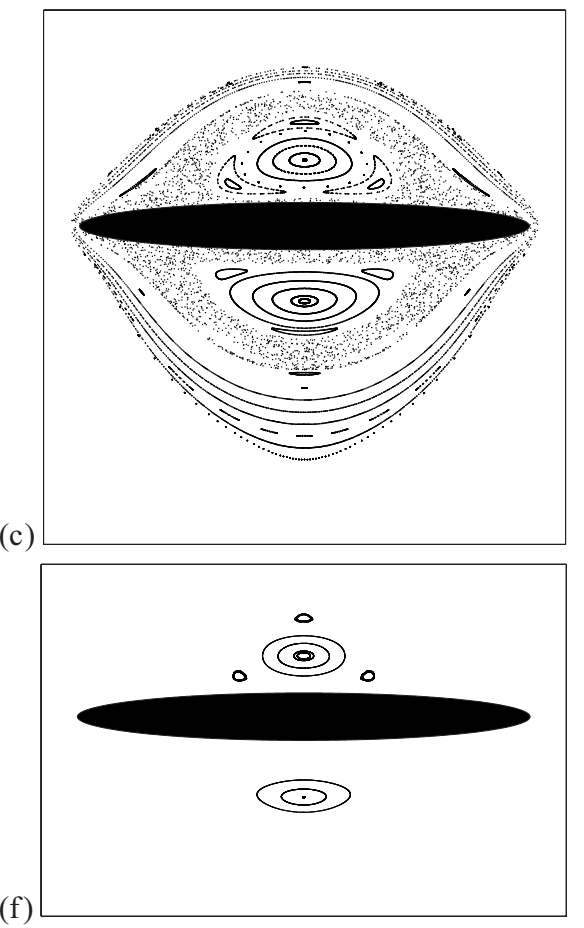

(f)
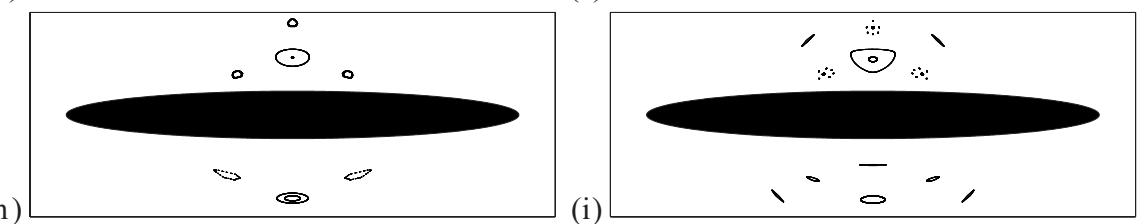

FIG. 2. Poincaré sections for a rotating and translating ellipse with $a=0.9$ in Eq. (1). The initial conditions are $z_{0}=\theta=0, \omega=1$ and (a) $v_{0}(0)=0.01,(\mathrm{~b}) 0.03$, (c) 0.05 , and so on up to (i) 0.19 . The atmosphere shrinks to just a few islands and eventually disappears as the initial translational velocity is increased.

tive and absolute tolerance both set to $10^{-8}$. It was verified that changing this by a factor of $10 \mathrm{did}$ not alter the results presented here. The kinetic energy of the system, Eq. (6), was conserved to one part in $10^{9}$ or better. This rivals the precision that one would have obtained by working with the exact solutions for the body motion.

Examples are shown in Fig. 2. A scatter plot generated in this way is a Poincaré section in the sense of dynamical systems theory with one difference: since particles that are eventually left behind are not part of the atmosphere, all points that such particles contribute to the Poincare section up to the end of the calculation are deleted. Thus, a particle that stays with the body for, say, 900 revolutions but is lost after revolution 901 is not counted as part of the atmosphere, and all the points it contributed to the Poincaré section are omitted from the final plot. To the extent that the calculations are picking out KAM surfaces, the particles in these regions must remain with the body forever. However, it is possible that a few of the particles contributing to the section plots will just stay with the body for a time longer than the integration period, but will eventually leave it.

We may choose our unit of length such that the airfoil size, say $R=|f(1)-f(0)|$, is 1 and our unit of time such that the initial angular velocity of rotation $\omega(0)=1$. The initially imposed linear velocity of translation along the major axis $v_{0}(0)$ is our main perturbation parameter. When $v_{0}(0)$ is small compared with $R \omega(0)$, in our chosen units $v_{0}(0) \ll 1$, we do find a region of fluid that follows the body forever. This region is the sought after atmosphere. It is made up of a number of regular "islands" embedded in a chaotic "sea" as is common for conservative dynamical systems of this kind. ${ }^{6}$ It should come as no surprise that the particle motion in the unsteady field of the moving body is, in general, chaotic. This is simply another manifestation of chaotic advection. ${ }^{10,11}$ The size, extent, and stability of the regular regions are the important new features. The diagrams shown in Fig. 2 correspond to the case where the ellipse is "neutrally buoyant," i.e., its mass equals the mass of the fluid it displaces.

We found that ellipses of large eccentricity are most effective in maintaining an atmosphere. We also found that in some cases the regular islands seem to be all that survives of an atmosphere, e.g., in Figs. 2(f)-2(i). In other cases there is an extensive "chaotic sea" as well, see Figs. 2(a)-2(e). One clearly sees how the chaotic orbits in Fig. 2(a) lie near the stagnation streamline in Fig. 1(a). As the perturbation strength is increased in Figs. 2(a)-2(c) this band becomes still wider. In these figures the chaotic sea appears to be delimited by a regular envelope. This envelope may be a KAM surface or it may be an island chain of very high period. In Figs. 2(d) and 2(e), for example, we seem to retain some chaotically moving particles even though we do not immediately see a delimiting KAM surface. It is, therefore, possible in these cases that the particles in the chaotic region 


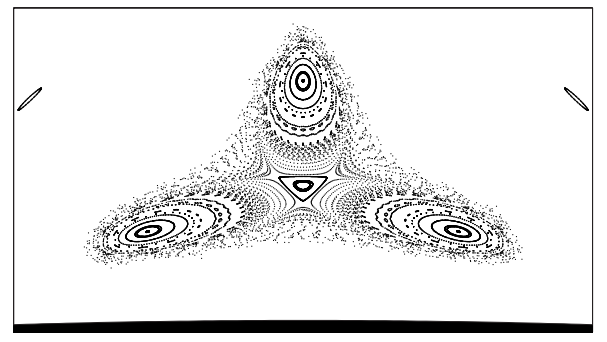

FIG. 3. Zoom showing structure of islands above ellipse for same initial conditions as in Fig. 2 except that $v_{0}(0)=0.2$.

will eventually exit the atmosphere and only the visible KAM islands will remain. Figure 3 shows the detailed structure of the remnant island above the ellipse when the initial velocity is $v_{0}(0)=0.2$.

Figure 4 probes a more mechanistic understanding of the atmosphere. Four individual particle trajectories are shown for the case $v_{0}(0)=0.05$, Fig. 2(c). Each panel shows the initial and final positions of the ellipse, its trajectory, and the trajectory of a judiciously chosen particle. The initial particle position and body center are shown as open circles, their end point as solid circles. (See the caption of Fig. 4 for precise starting positions.) We have shown the motion of the ellipse

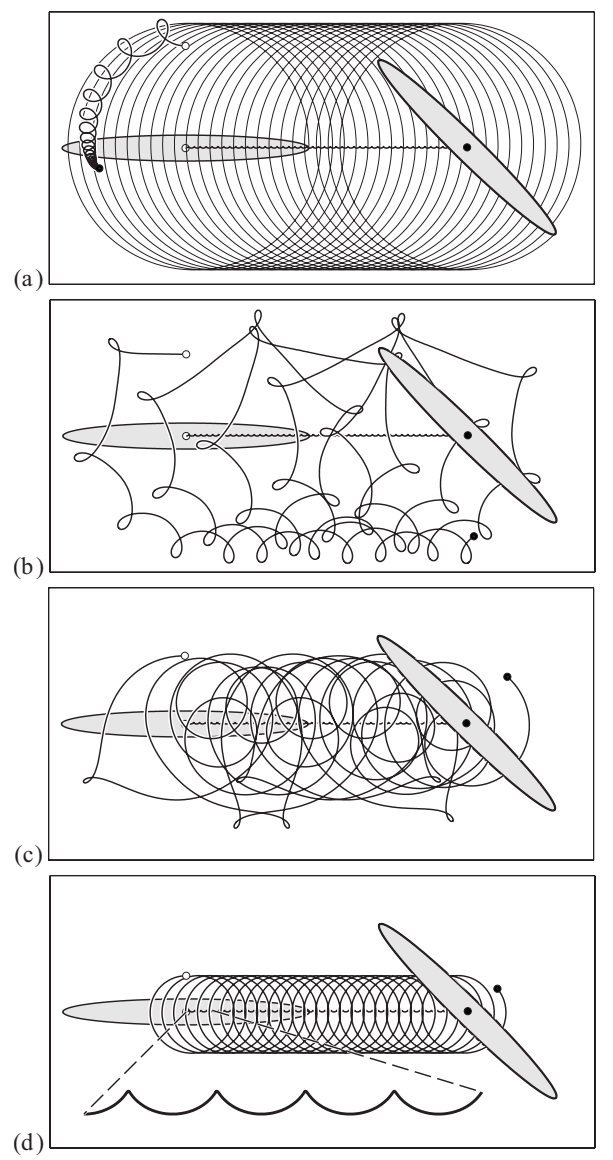

FIG. 4. Small piece of the trajectories of four particles calculated to generate the Poincaré section in Fig. 2(a). Initial (left) and final (right) ellipse positions and the trajectory of $z_{0}$ starting at $z_{0}=0$ are shown. The particle trajectories are started at (a) $z=1.5 i$, (b) $1.2 i$, (c) $i$, and (d) $0.53 i$. To illustrate the body motion we plot the tip trajectory in (a) and magnify a segment of the $z_{0}$ trajectory in (d).

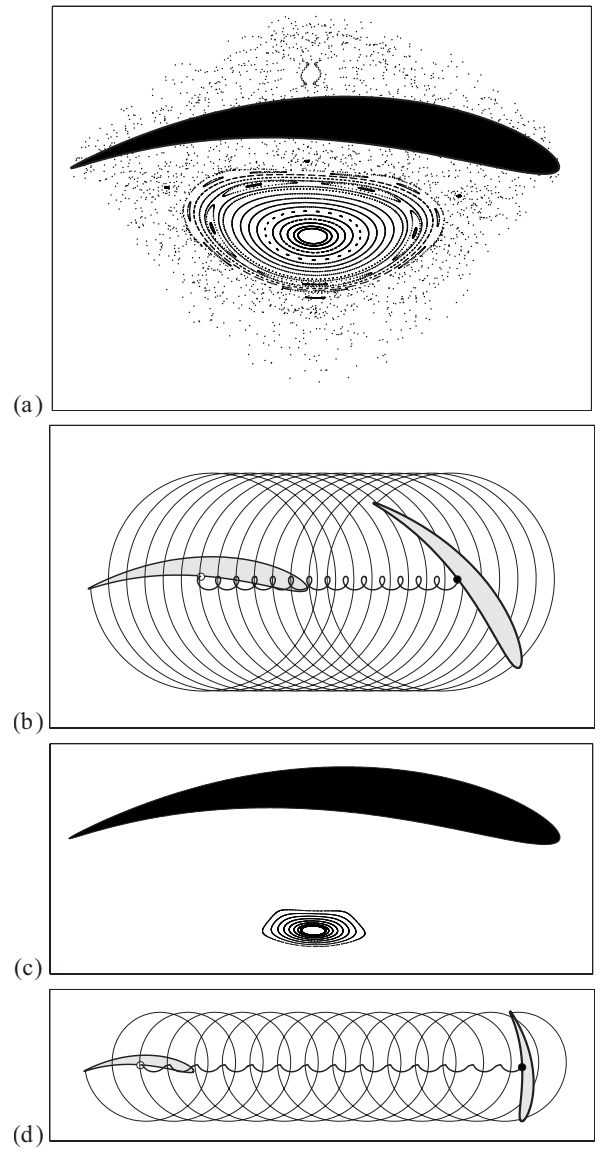

FIG. 5. Poincaré section showing atmosphere for perturbed rotating motion of a Joukowski airfoil. The airfoil is the same as shown in Fig. 1(b). The angular impulse is $L=1$ and the linear impulse $P$, which perturbs the steadily rotating motion, is (a) $P=0.05$ and (c) 0.15 . Body center and trailing edge trajectories corresponding to (a) and (b) are shown in (c) and (d), respectively.

in two ways in Fig. 4. First, in Fig. 4(a) we trace the trajectory of the tip of the ellipse as it progresses through the fluid. This clearly illustrates how the rotation of the body dominates its translation. In Fig. 4(d) we have magnified the trajectory of the center of the ellipse, highlighting the undulatory motion that it takes.

The particle in Fig. 4(a) is started too far away and is left behind by the ellipse in its motion toward the right. The particle in Fig. 4(b), on the other hand, is started close enough to become a part of the atmosphere. Its trajectory is regular with the particle receiving a "slap" from the ellipse at each loop. This slap moves the particle from one side of the ellipse to the other. In Fig. 4(c) we see a particle trajectory that is part of the chaotic atmosphere. The particle "seems unable to decide" whether it should orbit the ellipse or stay on one side of it. This sensitive decision at every turn of the ellipse is the mechanism leading to a chaotic particle track, and a chaotic region in the atmosphere in Fig. 2(c). Finally, in Fig. 4(d) we show a particle that remains on one side of the ellipse in a regular portion of the atmosphere. Comparing this particle trajectory with the trajectory of the tip of the ellipse in Fig. 4(a) vividly illustrates how the particle moves as if rigidly attached to the ellipse. The Poincaré sections of Fig. 2 provide a "template" of where the trapped particles 
originate and what region of space they occupy. The sample trajectories in Fig. 4 give examples of particle tracks that produce these Poincaré sections.

We were intrigued to explore if these results for ellipses would carry over to the motion of asymmetric objects such as the airfoil of Fig. 1(b). Figure 5 shows Poincaré sections for perturbations of the steadily rotating Joukowski airfoil of Fig. 1(b). Atmospheres are apparent in both cases but now the asymmetry of the body makes the regular island above the airfoil disappear much faster than the one below. We recall that in order to get a tight circular motion the mass of the airfoil has to be large compared with the mass of fluid displaced.

\section{CONCLUSIONS}

Documenting the existence of an atmosphere around a moving body without circulation around it in an ideal fluid is, so far as we know, a new observation. We have not given a complete parametric survey here but highlighted a typical case where an atmosphere does arise. Since atmospheres primarily arise for small translational velocity perturbations of a uniformly rotating body, one can argue that this is not a phenomenon one would readily observe in practical applications. Applications of the theory of motion of rigid bodies through ideal flow tend to concentrate on a state of steady translation of the body, such as an airplane wing in flight, and small perturbations around such a state. Without circulation about the body, or a trapped vortex, there is typically no atmosphere in this regime (in the inviscid case). The rotation-dominated regime where the atmosphere arises may be applicable to problems of objects falling through a fluid, in particular if the motion has the nature of tumbling. Falling plant seeds, or objects falling through the ocean, may thus carry fluid with them in an atmosphere. This transport mechanism may be of some interest in various contexts. Since it is inertially driven, it would probably occur trans- versely to a prevailing weak stratification. We also note in this connection that the problem of a falling plate ${ }^{9}$ has been linked to the problem of insect flight. Thus, it is possible that the beating wing of an insect also maintains a certain invariant volume of fluid around it even as the insect flies. We might conclude as Darwin ${ }^{5}$ did more than five decades ago: "In view of the great progress made in recent years in the study of the motion of real fluids, these matters may seem of secondary importance, but a knowledge of them does help in understanding some of the important features of the fluid motion."

\section{ACKNOWLEDGMENTS}

We thank Morten Brøns for comments and discussion and V. V. Meleshko for reminding us of the paper by Morton. This work is supported by a Niels Bohr Visiting Professorship at the Technical University of Denmark sponsored by the Danish National Research Foundation.

${ }^{1}$ Sir W. Thomson (Lord Kelvin), "On vortex atoms,” Proc. R. Soc. Edinburgh 6, 94 (1867).

${ }^{2}$ J. C. Maxwell, "On the displacement in a case of fluid motion," Proc. London Math. Soc. 1-3, 82 (1870).

${ }^{3}$ H. Lamb, Hydrodynamics, 6th ed. (Cambridge University Press, Cambridge, England, 1932).

${ }^{4} \mathrm{~W}$. B. Morton, "On the displacements of the particles and their paths in some cases of two-dimensional motion of a frictionless liquid," Proc. R. Soc. London, Ser. A 89, 106 (1913).

${ }^{5}$ Sir C. Darwin, "Note on hydrodynamics," Math. Proc. Cambridge Philos. Soc. 49, 342 (1953).

${ }^{6} \mathrm{M}$. Tabor, Chaos and Integrability in Nonlinear Dynamics: An Introduction (Wiley, New York, 1989).

${ }^{7}$ L. M. Milne-Thomson, Theoretical Hydrodynamics, 5th ed. (Dover, New York, 1968).

${ }^{8}$ J. Roenby and H. Aref, "Chaos in body-vortex interactions," Proc. R. Soc. London, Ser. A (in press).

${ }^{9}$ A. Andersen, U. Pesavento, and Z. J. Wang, "Unsteady aerodynamics of fluttering and tumbling plates," J. Fluid Mech. 541, 65 (2005).

${ }^{10}$ H. Aref, "Stirring by chaotic advection," J. Fluid Mech. 143, 1 (1984).

${ }^{11}$ H. Aref, "The development of chaotic advection," Phys. Fluids 14, 1315 (2002). 